\title{
Interkulturelle Öffnung: \\ Abbau von Zugangsbarrieren auf vier Ebenen
}

\section{DAVID RÜGER}

M.A. Soziale Arbeit, verwaltet die Professur Organisationsmanagement im Kontext regionaler Entwicklungsprozesse an der Fakultät Management, Soziale Arbeit, Bauen der Hochschule für angewandte Wissenschaft und Kunst (HAWK).

\section{MEHMET FATIH RÜZGAR}

M.A. Soziale Arbeit, ist wissenschaftlicher Mitarbeiter im Projekt Migrantische Ökonomie für ländliche Kommunen an der Fakultät Management, Soziale Arbeit, Bauen der Hochschule für angewandte Wissenschaft und Kunst (HAWK). Zugleich ist er Mitarbeiter des Kommunalen Integrationszentrums des Kreises Minden-Lübbecke.

http://www.hawk.de http://www.das-zukunftszentrum.de

\author{
Einrichtungen der Sozialen Arbeit können interkulturelle \\ Öffnungsprozesse auf verschiedenen Ebenen \\ anstoßen und vollziehen. Eine Schlüsselaufgabe \\ dabei ist die Vermeidung kultureller Kurzschlüsse.
}

\section{Warum Interkulturelle Öffnung?}

Lange Zeit gingen unterschiedliche Bundesregierungen in Deutschland davon aus, dass die in der zweiten Hälfte des 20. Jahrhundert angeworbenen Arbeitsmigrantinnen und -migranten irgendwann wieder in ihre Heimatländer zurückkehren würden. Aus diesem Grund wurden sie während dieser Zeit lediglich in (wohlfahrtsverbandlichen) Sondereinrichtungen versorgt (vgl. Handschuck 2008, 92ff; Kalpaka/Mecheril 2010, 79; Handschuck/Schröer 2012, 21ff).

Wie heute bekannt ist, hat sich die Annahme eines zeitlich befristeten Aufenthalts in zahlreichen Fällen nicht bewahrheitet. Im Gegenteil, Familien zogen nach oder wurden neugegründet und die zugewanderten Menschen wurden hier dauerhaft sesshaft. In den 1980 er- und 1990er-Jahren wuchs daher die politische Einsicht, öffentliche Einrichtungen sowohl strukturell als auch konzeptionell auf die gleichzeitige Anwesenheit von Einheimischen und Zugewanderten auszurichten und gerechte Zugangsbedingungen sowie demokratische Teilhabechancen für alle zu schaffen (vgl. Handschuck 2008, 92ff). Der Öffnungsprozess gegenüber inzwischen fast 20 Millionen Menschen mit (Arbeits-)Migrationsgeschichte (vgl. Statistisches Bundesamt 2018) ist seither ein wichtiges Thema auf der (sozial-) politischen Agenda der Bundesrepublik und wird es angesichts des inzwischen weitgehend akzeptierten Status als Einwanderungsland auch weiterhin bleiben.

\section{Was bedeutet Interkulturelle Öffnung?}

Interkulturelle Öffnung bezeichnet einen ganzheitlichen "Prozess der Organisationsentwicklung, der die Zugangsbarrieren für Migranten zu Bildung, Kultur und sozialen Diensten beseitigt « (Fischer 2013, 21).

\section{Zugangsbarrieren für Eingewanderte beseitigen}

Als Gegenstand und Ausgangspunkt Interkultureller Öffnung konzeptualisiert Fischer (2013) den Abbau von Zugangsbarrieren für Menschen mit Migrationsgeschichte. Gemeint sind damit sämtliche Faktoren, die sie von der Teilhabe an öffentlichen Gütern oder Dienstleistungen sowie politischer Mitsprache ausschließen. Folglich sind nicht Menschen mit Migrationsgeschichte Adressatinnen und Adressaten entsprechender Maßnahmen, sondern die Infrastrukturen und das einheimische Personal einer Einrichtung.

Zugangsbarrieren für Menschen mit Migrationsgeschichte resultieren in vielen Fällen aus der fehlenden Mehrsprachigkeit Einheimischer bzw. fehlenden muttersprachlichen Mitarbeitenden in allen Abteilungen einer Einrichtung. Denjenigen, denen deshalb zum Beispiel nicht mitgeteilt werden kann, an wen sie sich bezüglich bestimmter Unterstützungsleistungen wenden müssen, bleibt die Teilhabe folglich versperrt. Ebenso schließen bestimmte Deutungsund Handlungsmuster Menschen von 
Dienstleistungen und Mitspracheorganen aus. Fast alle kennen das Beispiel vom (religiös motivierten) verweigerten Handschlag eines Menschen mit Migrationsgeschichte, den Angestellte einer Einrichtung als Unfreundlichkeit deuten und deshalb zum Beispiel nur eine verkürzte Beratung anbieten. $\mathrm{Zu}$ letzt kann Ausschluss aus stereotypen Vorurteilen resultieren. Dies ist zum Beispiel der Fall, wenn einem jungen dunkelhäutigen Mann an der Tür einer Diskothek allein aufgrund seines Aussehens und seiner (vermeintlichen) Herkunft unterstellt wird, schnell aggressiv zu werden - und ihm nur aufgrund dieser Vorurteile der Zugang verwehrt wird.

\section{Ganzheitlicher Organisationsentwicklungsprozess}

Als relevante Ebenen für den Abbau von einrichtungsbezogenen Zugangsbarrieren gelten die Handlungsstrategien einer Einrichtung, ihre materielle Infrastruktur, ihr Personalbestand sowie ihre Kultur (vgl. Terkessidis 2010, 142ff). Zugleich weist Terkessidis darauf hin, dass Interkulturelle Öffnung nur gelingt, wenn auf allen vier Ebenen ineinander verzahnte Maßnahmen durchgeführt wird (vgl. ebd.).

Als Organisationskultur werden Werte und Normen verstanden, die sich eine Einrichtung selbst gibt und häufig in Form eines Leitbildes nach außen kommunizieren. Für die idealtypische Praxis Interkultureller Öffnung bedeutet dies, zu Beginn des Prozesses ein gemeinsames Leitbild zu entwickeln und so die Aufmerksamkeit und Bereitschaft der Mitarbeitenden und Führungskräfte für das Thema zu steigern.

Mit Personalbestand sind die Zusammensetzung eines Kollegiums sowie dessen hierarchische Binnenstruktur und die vorhandenen Wissensbestände der Angestellten gemeint. Neben der - oft leider einmaligen und damit zum symbolischen Akt verkürzten - Einstellung von Menschen mit sichtbarer Migrationsgeschichte erfordert fundierte Interkulturelle Öffnung auf der Ebene des Personalbestandes auch die Reflexion der Chancengerechtigkeit in Hinblick auf Chancengerechtigkeit betrieblicher Mitbestimmungsverfahren. Des Weiteren eignen sich institutionalisierte Fortbildungsstrategien, um Haltungen und
Handlungswissen der Mitarbeitenden zu entwickeln.

Im Hinblick auf die Architektur und Inneneinrichtung einer Einrichtung, also ihre materielle Infrastruktur, bedeutet Interkulturelle Öffnung im engen Sinne die Entwicklung mehrsprachiger Wegweiser und Informationsmaterialien. Darüber hinaus kann Interkulturelle Öffnung aber auch im weiten Sinne verstanden werden. Für die Soziale Arbeit bedeutet das konkret, während der Entwicklung von Maßnahmen die spezifischen Wünsche und Bedarfe der Zielgruppe mitzudenken und sie in Maßnahmen zu integrieren. Dabei ist es wichtig, die kulturspezifischen, sprachlichen und religiösen Besonderheiten zu berücksichtigen, um hierdurch Ansprache, Beteiligung und schließlich Zugang zu Angeboten sicherzustellen (vgl. Der Paritätische NRW 2014, 21f). Beispielsweise könnte der Zugang von dass dies nichts Neues für sie sei und Bildung in ihrer Familie schon immer einen hohen Stellenwert genoss. Terkessidis nennt solche Vorgänge »kulturelle Kurzschlüsse" (Terkessidis 2010, 118). Gemeint sind damit Prozesse, in denen entweder von der (vermeintlichen) natioethno-territorialen Herkunft einer Person auf bestimmte Handlungsweisen geschlossen wird oder umgekehrt eine bestimmte Handlungsweise vorschnell auf die (vermeintliche) Herkunft einer Person zurückgeführt wird. Für eine erfolgversprechende Interkulturelle Öffnung auf der Handlungsebene hat das Wissen über kulturelle Kurzschlüsse zwei Konsequenzen: Zum einen bedeuten es, davon auszugehen, dass Barrieren mehrdimensionale Ursachen haben und nicht allein auf eine Migrationserfahrung oder gar nur eine Migrationsgeschichte zurückgeführt werden können (vgl. Crenshaw 1989). Zum anderen be-

\section{"Da in Interkulturellen Öffnungsprozessen immer die Macht- und Privilegienfrage gestellt wird, erfordern sie stets die Bereitschaft Einheimischer zur Selbstkritik sowie} Anstrengungen, eigene, möglicherweise
verdeckt diskriminierende Denk- und Handlungsroutinen zu verändern."

Menschen mit Migrationsgeschichte in einem Begegnungszentrum erhöht werden, indem mehrsprachige Fernsehprogramme, Zeitschriften oder Bücher in den Bestand übernommen werden. Hierdurch kann wiederum eine aktive Teilnahme der Beteiligten an weiteren Maßnahmen bewirkt werden.

Handlungsstrategien sind Verfahren und Methoden, auf deren Grundlage Einrichtungen mit ihren Adressatinnen und Adressaten interagieren. Hinsichtlich ihres Öffnungspotenzials können sie unterschiedlich wirken, je nachdem wen sie adressieren. Wer beispielsweise pauschal alle Eltern mit Migrationsgeschichte über die Notwendigkeit eines Schulbesuchs ihrer Kinder informiert, wird relativ schnell auf Menschen treffen, die nett lächeln und entgegnen, deutet es, entsprechend mehrdimensionale Maßnahmen vorzuhalten. Gemeint sind damit zum Beispiel zwei parallele Maßnahmen sowohl für sprachlich, ökonomisch und aufenthaltsrechtlich benachteiligte Menschen als auch für ausschließlich sprachlich benachteiligte Menschen.

\section{Ganzheitlich oder kleine Schritte?}

Da in Interkulturellen Öffnungsprozessen immer die Macht- und Privilegienfrage gestellt wird (vgl. Mecheril/ Plößer 2018), erfordern sie stets die Bereitschaft Einheimischer zur Selbstkritik sowie Anstrengungen, eigene, möglicherweise verdeckt diskriminierende Denk- und Handlungsroutinen $\mathrm{zu}$ verändern. Interkulturelle Öffnung 
ist somit zwangsläufig konfliktbehaftet und angesichts dessen, dass Menschen ihre Privilegien eher behalten und nicht abgeben wollen, oft nur begrenzt realisierbar. Darüber hinaus fehlen mitunter personelle und finanzielle Ressourcen, um Einrichtungen unmittelbar auf allen vier Ebenen zu öffnen.

Zugleich tragen aber schon vereinzelte Öffnungsmaßnahmen dazu bei, dass Menschen barrierefreier an öffentlichen Ressourcen teilhaben können. Anstatt angesichts der großen Aufgabe überfordert aufzugeben, lohnt deshalb eine - kontinuierliche - Politik der kleinen Schritte (vgl. Stadt Herne o.J.). Hierbei kann helfen, verschiedene Perspektiven auf das Thema einzunehmen und zum Beispiel mit Hinweisen auf die positiven Auswirkungen Interkultureller Öffnung auf den Fachkräftemangel in der Bundesrepublik für ihre Notwendigkeit zu werben. Aus unserer Sicht handelt es sich dabei allerdings nur um einen positiven Nebeneffekt. In aller erster Linie geht es bei Interkultureller Öffnung um die Realisierung grundlegender Menschenrechte, Gerechtigkeit und Teilhabe für alle.

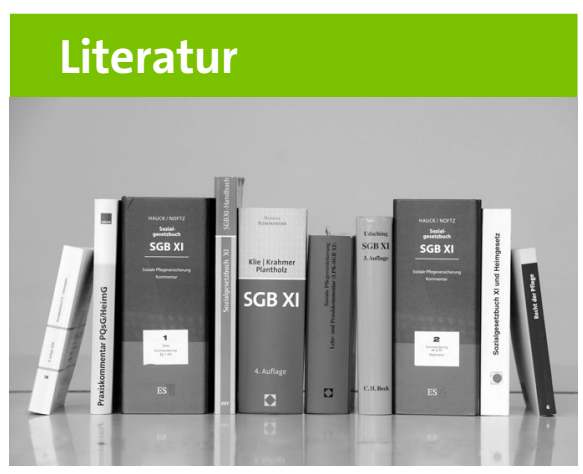

Crenshaw, Kimberle (1989): Demarginalizing the Intersection of Race and Sex: A Black Feminist Critique of Antidiscrimination Doctrine, Feminist Theory and Antiracist Politics. In: https:// chicagounbound.uchicago.edu/cgi/viewcontent. cgi?article $=1052 \&$ context=uclf (Zugriff: 22.04.2019) Der Paritätische NRW (2014): Leitfaden Interkulturelle Öffnung. Eine Arbeitshilfe zu interkulturellen Öffnungsprozessen in Mitgliedsorganisationen. In: https://www. paritaet-nrw.org/fileadmin/user_upload/ Bilder/05_Rat_und_Tat/03-Veroeffentlichungen/ Broschueren/Interkulturelle-Oeffnung-LeitfadenNRW.pdf (Zugriff: 13.05.2019)

Fischer, Veronika (2013): Gesellschaftliche Rahmenbedingungen für die Entwicklung migrationsbedingter Qualifikationserfordernisse. In: Fischer, Veronika u.a. (Hrsg.): Interkulturelle Kompetenz. Schwalbach: Debus Pädagogik, 11-30
Handschuck, Sabine (2008): Interkulturelle Qualitätsentwicklung im Sozialraum. Band 1. Konzeption eines Modellprojekts zur interkulturellen Orientierung und Öffnung von sozialen Einrichtungen. Augsburg: Ziel

Handschuck, Sabine/Schröer, Hubertus (2012): Interkulturelle Orientierung und Öffnung. Theoretische Grundlagen und 50 Aktivitäten zur Umsetzung. Augsburg: Ziel

\section{Kalpaka, Anita/Mecheril, Paul (2010):}

Interkulturell. Von spezifisch kulturalistischen Ansätzen zu allgemein reflexiven Perspektiven. In: Mecheril, Paul u.a. (Hrsg.): Migrationspädagogik. Weinheim und Basel: Beltz, 77-98

Mecheril, Paul/Plößer, Melanie (2018): Diversity und Soziale Arbeit. In: Otto, Hans-Uwe u.a. (Hrsg.): Handbuch Soziale Arbeit. 6., überarbeitete Auflage. München: Reinhardt, 283-292

Stadt Herne (o.J.): Handlungskonzept zur Interkulturelle Öffnung der Verwaltung. In: https://www.herne.de/Migration/_ IKOe/Handlungskonzept-IK\%C3\%96-_ endg\%C3\%BCltige-Fassung_.pdf.pdf (Zugriff: 13.05.2019)

Statistisches Bundesamt (Hrsg.) (2018): Pressemitteilung Nr. 282 vom 1. August 2018. In: https://www.destatis.de/DE/Presse/ Pressemitteilungen/2018/08/PD18_282_12511. html (Zugriff: 18.04.2019)

Terkessidis, Mark (2010): Interkultur. Berlin: Suhrkamp

\section{Eine empirische Studie über den Wandel der modernen Trauerkultur}

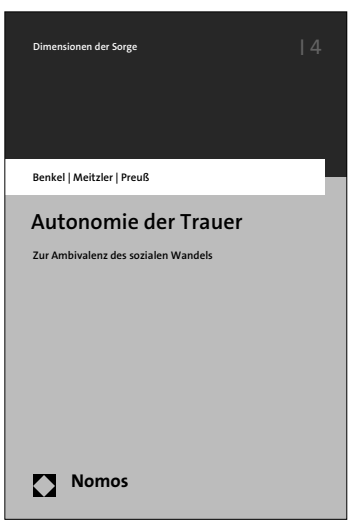

Autonomie der Trauer

Zur Ambivalenz des sozialen Wandels Von AkadR Dr. Thorsten Benkel, Matthias Meitzler und Dr. Dr. Dirk Preuß

2019, 220 S., brosch., 44,- $€$

ISBN 978-3-8487-6032-9

(Dimensionen der Sorge, Bd. 4)

nomos-shop.de/42060
Die Bestattungskultur ist im Umbruch. Während Handlungskonzepte rund um das Lebensende plural geworden sind, bleibt das Recht erstaunlich starr. Gegenwärtig etablieren sich Praktiken, bei denen die Asche Verstorbener abseits des Friedhofs aufbewahrt bzw. verstreut wird. Dies ist ein Wandel hin zur Autonomie der Trauer. 